\title{
Parametric Exponential Energy Decay for Dissipative Electron-Ion Plasma Waves *
}

\author{
Nikos Karachalios ${ }^{\dagger}$, Nikos M. Stavrakakis ${ }^{\ddagger}$ and Pavlos Xanthopoulos ${ }^{\ddagger}$
}

\begin{abstract}
We consider the following evolution system of Klein-Gordon-Schrödinger type

$$
\begin{aligned}
& i \psi_{t}+\kappa \psi_{x x}+i \alpha \psi=\phi \psi, x \in \Omega, t>0, \\
& \phi_{t t}-\phi_{x x}+\phi+\lambda \phi_{t}=-\operatorname{Re} \psi_{x}, x \in \Omega, t>0,
\end{aligned}
$$

satisfying the following initial and boundary conditions

$$
\begin{aligned}
& \psi(x, 0)=\psi_{0}(x), \phi(x, 0)=\phi_{0}(x), \phi_{t}(x, 0)=\phi_{1}(x), x \in \Omega, \\
& \psi(x, t)=\phi(x, t)=0, x \in \partial \Omega, t>0
\end{aligned}
$$

with $\kappa, \alpha, \lambda$ positive constants and $\Omega$ a bounded subset of $\mathbb{R}$. This system describes the nonlinear interaction between high frequency electron waves and low frequency ion plasma waves in a homogeneous magnetic field, adapted to model the UHH plasma heating scheme. The system focuses on the vital role of collisions, by considering the non-homogeneous polarization drift for the low frequency coupling. In Part I we set up the system, starting from first principles. In Part II we work out global existence and uniqueness of solutions and establish the necessary conditions for the system to manifest energy decay. In Part III the results are physically interpreted, providing a threshold of the effectiveness of $\mathrm{UHH}$, in terms of the plasma variables.
\end{abstract}

\section{Introduction and Motivation}

The aim of the present work is to study a model describing the Upper Hybrid Heating (UHH) scheme for plasmas in fusion devices. UHH is the dominant branch of the general Electron Cyclotron Resonance Heating (ECRH) scheme, which, for tokamaks and stellarators, constitutes a basic method of plasma build-up and

\footnotetext{
${ }^{*}$ Keywords and Phrases: Klein-Gordon - Schrödinger system, Electron-Ion Plasma Waves, ECRH Plasma Heating, Dissipation, Global Existence, Uniqueness, Energy Decay.

AMS Subject Classification: 35B30, 35B40, 35L70, 35L05, 35Q55

To appear in: Z. Angew. Math. Phys.
} 
heating (the W7-X experiment, currently under construction, will be equipped with an ECRH power of $10 \mathrm{MW}$ ). Moreover, ECRH is an attractive method to study transport mechanisms, since it allows for a very localised power deposition, thus influencing temperature and current profiles ([21]).

The UHH scheme consists in injecting electromagnetic waves in the range 100-200 GHz, from the high field side towards the core of the device. Within this frequency range, the incident wave takes on the character of a longitudinal oscillation for the resonant electrons, which become highly energetic.

With respect to the physical mechanism involved in the energy damping of the waves, UHH comprises two stages:

(i) Collisionless damping. The energy of the waves is transferred to the resonant electrons, through collisionless mechanisms, e.g. Landau damping. Subsequently, the electrons gain excessive kinetic energy, thus heated.

(ii) Collisional damping. The excessive electron energy is distributed over electrons and non-resonant ions, through Coulomb collisions, producing bulk heating of the plasma (equipartition).

Collisional damping is very crucial for the success of UHH. If collisions are infrequent, non-thermal distributions will occur, which may result in a reduction in the power delivered to the plasma ([22]). Therefore, it is important to determine the operational conditions for the device, under which UHH becomes effective, namely the collisions manage to distribute the excessive electron energy over the species at an exponential rate. To the best of our knowledge, this task is not as yet undertaken.

Concerning the physical context, we investigate the nonlinear interaction between high frequency electron waves and low frequency ion plasma waves in a homogeneous magnetic field. This phenomenon arises, once a high frequency electric field with low frequency amplitude propagates through the plasma.

The celebrated Zakharov system (see [23] and [4], [5], [7], [9], [15], [17], [19], for the well-posedness and properties of solutions), is highly successful in a multitude of applications, such as laser fusion, electron beam fusion, solar radio bursts etc. ([18]). However, regarding the study of UHH, the Zakharov system may not be implemented for the following reasons:

(i) It does not consider the effect of collisions. Therefore, it can only describe the collisionless part of the damping.

(ii) It is indifferent to the presence of a dc magnetic field, due to the nature of the ponderomotive force. Therefore, perpendicular waves, peculiar to UHH heating, cannot be modelled.

In order to overcome these shortcomings we study the effect of the spacetime varying electric field on the ion channel. Specifically, we consider the drift motion of the ions caused by the time variation of the electric field, namely the polarization drift.

However, the space variation of the electric field is included in the polariza- 
tion drift. Indeed, it turns out that the contribution of this effect to the system of equations involves the space derivative of the electric field. In this respect, we may talk about a non-homogeneous polarization drift (note that a homogeneous time-varying field is sufficient for the standard polarization drift to occur). This drift induces a polarization current, which plays the role of the low frequency coupling between ions and electrons.

This consideration is beneficial in several ways. The polarization current allows for a straightforward treatment of the collision term in the momentum equations. In this respect, we retain full two-fluid dynamics, by keeping both continuity equations. Finally, the dc magnetic field is automatically accounted for, since the polarization drift is a secondary effect to the $\vec{E} \times \vec{B}$ drift flow ([16]).

In Part I, we accomplish the physical analysis of the problem and set up the system of equations. For this, the standard fluid equations are considered, with the collision term modelled as in [11]. It turns out that the system belongs to the dissipative Klein-Gordon Schrödinger (KGS) class. Problems of this kind have been examined with respect to the existence of solutions and global attractor in both bounded (see e.g. [1], [8], [10]), and unbounded domains of $\mathbb{R}^{n}$, for $n \leq 3$ (see e.g. [2], [13]).

Our main task, however, is to investigate the parametric energy decay for the system. Specifically, we seek necessary conditions, dependent on the parameters of the system, so that energy decay occurs at an exponential rate. This ensures that, under specific plasma conditions, the energy of the coupled ion-electron wave is effectively dissipated to the plasma. In this context, we also mention that in [3], a similar treatment is carried out for another KGS problem, although the issue of parameter dependence is not addressed.

In the first half of Part II we prove global existence and uniqueness for the solutions. In the last half, we derive necessary conditions for the parametric energy decay.

Part III deals with the physical interpretation of the results. In particular, the necessary conditions extracted in Part II are transformed into a threshold of the effectiveness of the heating scheme, in terms of the plasma variables (density, ion and electron temperatures) as well as the magnetic field. The model predicts that the scheme becomes extremely effective under high-density conditions, as in the very promising density-limit shots (see e.g. [14]), where also the temperature assumes relatively low values.

\section{Part I: Physical Setup}

The derivation of the system of equations is based on standard heuristic arguments, also used for the Zakharov system (see e.g. [16]). The particulars of our setup are as follows: 
We assume an oscillating electric field in the upper hybrid frequency range, producing resonant electrons. However, the polarization drift, as a low frequency mechanism, couples electrons with ions and energy is transferred to the latter, through collisions.

Concerning geometry, the dc magnetic field occupies the $\hat{z}$ direction, whereas the electric field is oriented in the $\hat{x}$ direction. Therefore, on the $x y$ plane, two distinct motions take place: (i) the $\vec{E} \times \vec{B}$ drift (in the $-\hat{y}$ direction) and (ii) the polarization drift (in the $\hat{x}$ direction). Both motions are taken into account in the corresponding momentum equations. Therefore, even though the mathematical system turns out to be 1-D, the following setup effectively describes the 3-D physical dynamics.

\subsection{Configuration}

We consider a homogeneous, constant magnetic field $\vec{B}=B_{0} \hat{z}$. The high frequency electric field has the form

$$
\vec{E}(x, t)=\tilde{E}(x, t) \exp \left(-i \omega_{u h} t\right) \hat{x}
$$

with

$$
\tilde{E}(x, t)=E_{0}(x) \exp (-i \omega t) \cos (k x)+i E_{1}(x),
$$

where $\omega_{p}$ is the plasma frequency, $\omega_{e}$ is the electron gyrofrequency, $\omega_{u h}=\sqrt{\omega_{p}^{2}+\omega_{e}^{2}}$ is the upper-hybrid frequency and $\omega \ll \omega_{i}$, where $\omega_{i}$ is the ion gyrofrequency. We adopt the following frequency ordering

$$
\tau_{H}^{-1} \leq \omega \ll \omega_{i} \ll \omega_{e}<\omega_{p}<\omega_{u h},
$$

where $\tau_{H}^{-1}$ is associated to the ambipolar potential and corresponds to the low frequency. In view of this ordering, note that the amplitude $\tilde{E}$ lies in the low frequency range, as well. Moreover, it is useful to break up the plasma variables, namely densities and temperatures (we do not treat heat dynamics), as follows

$$
\begin{array}{cl}
n_{i}=n_{0}+n_{i}^{L}, & n_{e}=n_{0}+n_{e}^{L}+n_{e}^{H}, \\
v_{i, x}=v_{i, x}^{L}+v_{i}^{p o l}, & v_{e, x}=v_{e, x}^{L}+v_{e, x}^{p o l}+v_{e, x}^{H}, \\
v_{i, y}=v_{i, y}^{L}, & v_{e, y}=v_{e, y}^{L}+v_{e, y}^{H} .
\end{array}
$$

In the above scheme, $n_{0}$ stands for the background density, which is common for ions and electrons due to quasineutrality. We also assume quasineutrality for the low frequency densities, i.e.,

$$
n_{i}^{L}=n_{e}^{L}=n^{L} \ll n_{0} .
$$


Now, since it holds $\omega \ll \omega_{i}$, the expression for the polarization drift velocity reads as follows $([6])$

$$
{\overrightarrow{v_{k}}}^{p o l}=\frac{c}{\omega_{k} B_{0}} \frac{d}{d t} \tilde{E}_{r} \hat{x}, \quad k=e, i,
$$

where $\tilde{E}_{r}$ denotes the real part of the amplitude. The following estimate

$$
\frac{\left|v_{e}^{p o l}\right|}{v_{i}^{p o l}} \propto \frac{m_{e}}{m_{i}} \ll 1
$$

implies that the polarization drift mainly affects the ions. On the other hand, it produces a polarization current, which has the form

$$
\vec{J}^{p o l}=n_{0} e\left({\overrightarrow{v_{i}}}^{p o l}-{\overrightarrow{v_{e}}}^{p o l}\right) \cong \frac{n_{0} c^{2} m_{i}}{B_{0}^{2}} \frac{d}{d t} \tilde{E}_{r} \hat{x}
$$

\subsection{Ion Dynamics}

In order to treat the effect of the polarization drift, we write the continuity equation in the following form

$$
\frac{\partial}{\partial t} n^{L}+n_{0} \frac{\partial}{\partial x} v_{i, x}^{L}+n_{0} \frac{\partial}{\partial x} v_{i}^{p o l}=0
$$

i.e., we take the relevant contribution out of the total flux. The momentum equation in the $\hat{x}$ direction including collisions reads

$$
\begin{aligned}
m_{i} n_{0} \frac{\partial}{\partial t} v_{i, x}^{L}= & -\gamma_{i} T_{i} \frac{\partial}{\partial x} n^{L}+e n_{0} E^{L}+\frac{e n_{0}}{c} v_{i, y}^{L} B_{0} \\
& +n_{0} m_{e} \nu_{e i}\left(v_{e, x}^{L}-v_{i, x}^{L}\right),
\end{aligned}
$$

where $E^{L}$ stands for the ambipolar electric field, for which Poisson's law, in view of $(2.2)$, gives

$$
\frac{\partial}{\partial x} E^{L}=4 \pi e\left(n_{i}^{L}-n_{e}^{L}\right)=0 .
$$

Our intent is to differentiate equation (2.6) with respect to $x$, therefore, we successively specify the various contributions. Starting with the Lorentz term, we adopt a minimal momentum equation in the $\hat{y}$ direction, as

$$
\frac{\partial}{\partial t} v_{i, y}^{L}=-\omega_{i} v_{i, x}^{L}
$$

We differentiate (2.8) with respect to $x$ and using (2.5) we get

$$
\frac{\partial}{\partial t} \frac{\partial}{\partial x} v_{i, y}^{L}=-\omega_{i} \frac{\partial}{\partial x} v_{i, x}^{L}=\frac{\omega_{i}}{n_{0}} \frac{\partial}{\partial t} n^{L}+\omega_{i} \frac{\partial}{\partial x} v_{i}^{p o l}
$$


from which, with the aid of (2.3), it follows

$$
\frac{\partial}{\partial x} v_{i, y}^{L}=\omega_{i} \frac{n^{L}}{n_{0}}+\frac{c}{B_{0}} \frac{\partial}{\partial x} \tilde{E}_{r}
$$

Next, we differentiate the collision term $K=n_{0} m_{e} \nu_{e i}\left(v_{e, x}^{L}-v_{i, x}^{L}\right)$ with respect to $x$. Since the electron velocity is involved, we take the low frequency part of the electron continuity equation,

$$
\frac{\partial}{\partial t} n^{L}+n_{0} \frac{\partial}{\partial x} v_{e, x}^{L}+n_{0} \frac{\partial}{\partial x} v_{e}^{p o l}=0
$$

Now, along with (2.2), (2.4) and (2.5), we obtain the expression for the collision term via the polarization current, as

$$
\begin{aligned}
\frac{\partial}{\partial x} K=n_{0} m_{e} \nu_{e i} \frac{\partial}{\partial x}\left(v_{e, x}^{L}-v_{i, x}^{L}\right) & =\frac{m_{e} \nu_{e i}}{e} \frac{\partial}{\partial x} J^{p o l} \\
& =\frac{n_{0} c^{2} m_{i} m_{e} \nu_{e i}}{e B_{0}^{2}} \frac{\partial}{\partial t} \frac{\partial}{\partial x} \tilde{E}_{r}
\end{aligned}
$$

Now, differentiate (2.6) with respect to $x$, also using (2.7), (2.9) and (2.10), to get

$$
\begin{aligned}
\frac{\partial}{\partial t} \frac{\partial}{\partial x} v_{i, x}^{L}= & -\frac{\gamma_{i} T_{i}}{m_{i} n_{0}} \frac{\partial^{2}}{\partial x^{2}} n^{L}+\omega_{i}^{2} \frac{n^{L}}{n_{0}}+\frac{e}{m_{i}} \frac{\partial}{\partial x} \tilde{E}_{r} \\
& +\frac{c^{2} m_{e} \nu_{e i}}{e B_{0}^{2}} \frac{\partial}{\partial t} \frac{\partial}{\partial x} \tilde{E}_{r} .
\end{aligned}
$$

Note here that $\frac{\partial}{\partial x} \tilde{E}_{r}$, as appears in equation (2.11), is not zero, since $\tilde{E}_{r}$ is not ambipolar. This follows from the charge separation created by the polarization drift. In fact, since $\tau_{H}^{-1} \leq \omega$, Poisson's law gives

$$
\frac{\partial}{\partial x} \tilde{E}_{r} \cong 4 \pi e n^{L} .
$$

This expression will be substituted into the collision term of (2.11). However, we leave the other occurrence of the term $\frac{\partial}{\partial x} \tilde{E}_{r}$ intact to take care of the coupling with the electron dynamics. Finally, considering (2.5), we approximate the left side of (2.11) as

$$
\frac{\partial}{\partial t} \frac{\partial}{\partial x} v_{i, x}^{L} \cong-\frac{1}{n_{0}} \frac{\partial^{2}}{\partial t^{2}} n^{L} .
$$

In view of (2.12) and (2.13), equation (2.11) takes the final form

$$
\frac{\partial^{2}}{\partial t^{2}} n^{L}-\gamma_{i} v_{t h, i}^{2} \frac{\partial^{2}}{\partial x^{2}} n^{L}+\omega_{i}^{2} n^{L}+\nu_{e i} \frac{\omega_{p}^{2}}{\omega_{e}^{2}} \frac{\partial}{\partial t} n^{L}=-\frac{e n_{0}}{m_{i}} \frac{\partial}{\partial x} \tilde{E}_{r} .
$$


Remark 2.1 Note that the unusual form of the right side of equation (2.14), as compared to the corresponding Zakharov equation, is a consequence of the different low frequency coupling that we consider, i.e., the polarization drift instead of the ponderomotive force.

\subsection{Electron Dynamics}

We write down the electron fluid equations corresponding to the high frequency. In this respect, note that products of high frequency quantities are considered to be of higher order and thus suppressed. The continuity equation reads

$$
\frac{\partial}{\partial t} n_{e}^{H}+\frac{\partial}{\partial x}\left[\left(n_{0}+n^{L}\right) v_{e, x}^{H}\right]=0
$$

The momentum equation in the $\hat{x}$ direction, including collisions, is

$$
m_{e} n_{0} \frac{\partial}{\partial t} v_{e, x}^{H}=-\gamma_{e} T_{e} \frac{\partial}{\partial x} n_{e}^{H}-e n_{0} E-\frac{e n_{0}}{c} v_{e, y}^{H} B_{0}-m_{e} n_{0} \nu_{e i} v_{e, x}^{H} .
$$

Note that the high frequency part of the collision term is dominated by the electrons. In the following, we express each term of equation (2.16) via the electric field $E$. As this affects mainly the electrons, it induces charge separation, therefore Poisson's law gives (compare to (2.12))

$$
\frac{\partial}{\partial x} E=-4 \pi e n_{e}^{H} .
$$

Now, differentiate (2.15) with respect to $t$ to get

$$
\frac{\partial^{2}}{\partial t^{2}} n_{e}^{H}+\frac{\partial}{\partial x}\left[\left(n_{0}+n^{L}\right) \frac{\partial}{\partial t} v_{e, x}^{H}\right]=0 .
$$

From (2.17) and (2.18) we have

$$
\frac{\partial^{2}}{\partial t^{2}} E-4 \pi e\left(n_{0}+n^{L}\right) \frac{\partial}{\partial t} v_{e, x}^{H}=0 .
$$

Also, taking the derivative of (2.17) with respect to $x$ we get

$$
\frac{\partial}{\partial x} n_{e}^{H}=-\frac{1}{4 \pi e} \frac{\partial^{2}}{\partial x^{2}} E .
$$

Next, similar to (2.8), the momentum equation in the $\hat{y}$ direction reads

$$
\frac{\partial}{\partial t} v_{e, y}^{H}=\left|\omega_{e}\right| v_{e, x}^{H}
$$


Now, Ampére's law, for the homogeneous magnetic field, gives

$$
4 \pi J_{x}=-\frac{\partial}{\partial t} E
$$

with

$$
J_{x}=-e n_{0} v_{e, x}^{H}
$$

Therefore,

$$
\frac{\partial}{\partial t} E=4 \pi e n_{0} v_{e, x}^{H} .
$$

In order to obtain the expression for $v_{e, y}^{H}$, we take the derivative of (2.21) with respect to $x$ and use equations (2.15) and (2.17), to get

$$
\frac{\partial}{\partial t} \frac{\partial}{\partial x} v_{e, y}^{H}=\left|\omega_{e}\right| \frac{\partial}{\partial x} v_{e, x}^{H} \cong-\frac{\left|\omega_{e}\right|}{n_{0}} \frac{\partial}{\partial t} n_{e}^{H},
$$

which implies that

$$
\frac{\partial}{\partial x} v_{e, y}^{H}=-\frac{\left|\omega_{e}\right|}{n_{0}} n_{e}^{H}=\frac{\left|\omega_{e}\right|}{4 \pi e n_{0}} \frac{\partial}{\partial x} E .
$$

From the last equation we obtain

$$
v_{e, y}^{H}=\frac{\left|\omega_{e}\right|}{4 \pi e n_{0}} E .
$$

With the aid of the expressions (2.19), (2.20) and (2.23), equation (2.16) becomes

$$
\begin{aligned}
\frac{\partial^{2}}{\partial t^{2}} E & -\left(1+\frac{n^{L}}{n_{0}}\right) \frac{\gamma_{e} T_{e}}{m_{e}} \frac{\partial^{2}}{\partial x^{2}} E+\omega_{p}^{2}\left(1+\frac{n^{L}}{n_{0}}\right) E \\
& +\omega_{e}^{2}\left(1+\frac{n^{L}}{n_{0}}\right) E+\nu_{e i}\left(1+\frac{n^{L}}{n_{0}}\right) \frac{\partial}{\partial t} E=0 .
\end{aligned}
$$

Now, we assume that $1+\frac{n^{L}}{n_{0}} \cong 1$. Note, however, that this approximation breaks down once multiplied by $\omega_{p}^{2}$, which contains $n_{0}$ as well. We have

$$
\frac{\partial^{2}}{\partial t^{2}} E-\frac{\gamma_{e} T_{e}}{m_{e}} \frac{\partial^{2}}{\partial x^{2}} E+\omega_{u h}^{2} E+\nu_{e i} \frac{\partial}{\partial t} E=-\omega_{p}^{2} \frac{n^{L}}{n_{0}} E .
$$

Finally, we apply expression (2.1) to equation (2.25), also making use of the approximation

$$
\left|\frac{\partial}{\partial t} \tilde{E}\right| \ll\left|\omega_{u h} \tilde{E}\right|
$$

which follows from the frequency ordering. The resulting equation is

$$
2 i \omega_{u h} \frac{\partial}{\partial t} \tilde{E}+\gamma_{e} v_{t h, e}^{2} \frac{\partial^{2}}{\partial x^{2}} \tilde{E}+i \omega_{u h} \nu_{e i} \tilde{E}=\omega_{p}^{2} \frac{n^{L}}{n_{0}} \tilde{E} .
$$




\section{Part II: Mathematical Analysis}

\subsection{Global Existence}

In this part, we treat the system derived in the previous section, namely equations (2.14) and (2.26) in dimensionless form (details are given in Part III). In particular, the variable $\psi$ stands for the dimensionless low frequency electric field, whereas the (real) variable $\phi$ denotes the dimensionless low frequency density. Considering $\Omega$ a bounded domain of $\mathbb{R}$, the set of equations reads

$$
\begin{array}{r}
i \psi_{t}+\kappa \psi_{x x}+i \alpha \psi=\phi \psi, \quad x \in \Omega, \quad t>0, \\
\phi_{t t}-\phi_{x x}+\phi+\lambda \phi_{t}=-\operatorname{Re} \psi_{x}, \quad x \in \Omega, \quad t>0,
\end{array}
$$

with $\kappa, \alpha, \lambda>0$. The initial and boundary conditions are

$$
\begin{aligned}
\psi(x, 0) & =\psi_{0}(x), \phi(x, 0)=\phi_{0}(x), \phi_{t}(x, 0)=\phi_{1}(x), \quad x \in \Omega \\
\psi(x, t) & =\phi(x, t)=0, \quad x \in \partial \Omega, \quad t>0 .
\end{aligned}
$$

For completeness we state two well known results that will be frequently used in the following ([20], [24]).

Lemma 3.1 (Gagliardo-Nirenberg Inequality) Let $1 \leq p, q, r \leq \infty, j$ an integer such that, $0 \leq j \leq m$ and $j / m \leq \theta \leq 1$. Then, for any bounded domain in $\mathbb{R}^{n}$, the following inequality is true

$$
\left\|D^{j} u\right\|_{p} \leq \mathrm{const} \mid\|u\|_{q}^{1-\theta}\left\|D^{m} u\right\|_{r}^{\theta}, \quad u \in L^{q} \cap W^{m, r}(\Omega), \quad \Omega \subseteq \mathbb{R}
$$

where the constants satisfy the relation

$$
\frac{1}{p}=\frac{j}{n}+\theta\left(\frac{1}{r}-\frac{m}{n}\right)+\frac{1-\theta}{q} .
$$

Lemma 3.2 (Gronwall Inequality) Let $g, h, y$ be three positive locally integrable functions in the interval $t_{0}<t<\infty$, for some $t_{0}>0$, which satisfy the differential inequality

$$
\frac{d y}{d t} \leq g y+h, \text { for all } t \geq t_{0} .
$$

Then, the following uniform estimate for the function $y(t)$ is valid

$$
y(t) \leq y\left(t_{0}\right) \exp \left(\int_{t_{0}}^{t} g(\tau) d \tau\right)+\int_{t_{0}}^{t} h(s) \exp \left(\int_{s}^{t} g(\tau) d \tau\right) d s, \quad t \geq t_{0} .
$$

For the sake of brevity, we highlight only the most important relations. In what follows, we denote the time derivative with a prime. Also, $\|\cdot\|$ stands for the $L^{2}(\Omega)$ norm, whereas $\int d x$ denotes integration over the domain $\Omega$. Finally, $C$ 
is a generic symbol for any positive constant. We begin by postulating the real variable $\theta=\phi^{\prime}+\delta \phi$, where $\delta$ is an auxiliary positive constant to be specified later. Now, the system (3.1)-(3.4) takes the form

$$
\begin{aligned}
i \psi^{\prime}+\kappa \psi_{x x}+i \alpha \psi & =\phi \psi, \quad x \in \Omega, \quad t>0, \\
\phi^{\prime}+\delta \phi & =\theta, \quad x \in \Omega, \quad t>0, \\
\theta^{\prime}+(\lambda-\delta) \theta-\phi_{x x}+(1-\delta(\lambda-\delta)) \phi & =-\operatorname{Re} \psi_{x}, \quad x \in \Omega, \quad t>0 .
\end{aligned}
$$

Also the initial and boundary conditions take the form

$$
\begin{aligned}
& \psi(x, 0)=\psi_{0}(x), \phi(x, 0)=\phi_{0}(x), \theta(x, 0)=\theta_{0}(x), \quad x \in \Omega, \\
& \psi(x, t)=\phi(x, t)=0, \quad x \in \partial \Omega, \quad t>0 .
\end{aligned}
$$

The rest of this paragraph contains the a priori estimates which establish the existence of a global solution. Note, however, that due to the first order derivative on the right hand side of the equation (3.7), working in a dual space setting is not helpful.

First Estimate: We start with the derivation of a priori estimates for the solutions $(\psi, \phi, \theta) \in H_{0}^{1}(\Omega) \times H_{0}^{1}(\Omega) \times L^{2}(\Omega)$, assuming that there exists a constant $R>0$ such that $\left\|\left(\psi_{0}, \phi_{0}, \theta_{0}\right)\right\|_{H_{0}^{1} \times H_{0}^{1} \times L^{2}} \leq R$. Multiplying (3.5) by $\bar{\psi}$, integrating over $\Omega$ and taking the imaginary part, yields

$$
\frac{1}{2} \frac{d}{d t}\|\psi\|^{2}+\alpha\|\psi\|^{2}=0
$$

Now, applying Gronwall's inequality (Lemma 3.2), we obtain

$$
\|\psi(t)\| \leq\|\psi(0)\| e^{-2 \alpha t}, \quad t \geq 0
$$

Therefore, we get the estimate

$$
\|\psi(t)\| \leq R, \quad t \geq 0 .
$$

Next, we multiply (3.5) by $-\bar{\psi}^{\prime}$, integrate and take the real part as

$$
\frac{1}{2} \frac{d}{d t} \kappa\left\|\psi_{x}\right\|^{2}+\alpha \operatorname{Im} \int \psi \bar{\psi}^{\prime} d x=-\operatorname{Re} \int \phi \psi \bar{\psi}^{\prime} d x .
$$

Using the above transformation, the right hand side takes the following form

$$
-\operatorname{Re} \int \phi \psi \bar{\psi}^{\prime} d x=-\frac{1}{2} \frac{d}{d t} \int \phi|\psi|^{2} d x+\frac{1}{2} \int \theta|\psi|^{2} d x-\frac{\delta}{2} \int \phi|\psi|^{2} d x .
$$

Also, we have

$$
\alpha \operatorname{Im} \int \psi \bar{\psi}^{\prime} d x=\kappa \alpha\left\|\psi_{x}\right\|^{2}+\alpha \int \phi|\psi|^{2} d x
$$


Therefore, equation (3.13) takes the form

$$
\begin{aligned}
\frac{1}{2} \frac{d}{d t}\left(\kappa\left\|\psi_{x}\right\|^{2}+\int \phi|\psi|^{2} d x\right) & +\kappa \alpha|| \psi_{x} \|^{2}+\left(\alpha+\frac{\delta}{2}\right) \int \phi|\psi|^{2} d x \\
& =\frac{1}{2} \int \theta|\psi|^{2} d x .
\end{aligned}
$$

We proceed with equation (3.7), multiplying by $\theta$ and integrating to get

$$
\begin{aligned}
\frac{1}{2} \frac{d}{d t}\|\theta\|^{2} & +(\lambda-\delta)\|\theta\|^{2}-\int \phi_{x x} \theta d x+(1-\delta(\lambda-\delta)) \int \phi \theta d x \\
& =-\operatorname{Re} \int \psi_{x} \theta d x .
\end{aligned}
$$

Substituting $\theta$ from equation (3.6) into $-\int \phi_{x x} \theta d x$ and $(1-\delta(\lambda-\delta)) \int \phi \theta d x$, equation (3.15) becomes

$$
\begin{aligned}
\frac{1}{2} \frac{d}{d t}\left(\|\theta\|^{2}+\left\|\phi_{x}\right\|^{2}+(1-\delta(\lambda-\delta))\|\phi\|^{2}\right) & +(\lambda-\delta)\|\theta\|^{2} \\
+\delta\left\|\phi_{x}\right\|^{2}+\delta(1-\delta(\lambda-\delta))\|\phi\|^{2} & =-\operatorname{Re} \int \psi_{x} \theta d x .
\end{aligned}
$$

We introduce the quantities

$$
F_{1}:=\kappa\left\|\psi_{x}\right\|^{2}+\int \phi|\psi|^{2} d x+\|\theta\|^{2}+\left\|\phi_{x}\right\|^{2}+(1-\delta(\lambda-\delta))\|\phi\|^{2}
$$

and

$$
\begin{aligned}
G_{1}:= & +(\delta-2 \kappa \alpha)\left\|\psi_{x}\right\|^{2}-2 \alpha \int \phi|\psi|^{2} d x+(3 \delta-2 \lambda)\|\theta\|^{2} \\
& -\delta(1-\delta(\lambda-\delta))\|\phi\|^{2}-\delta\left\|\phi_{x}\right\|^{2}+\int \theta|\psi|^{2} d x-2 \operatorname{Re} \int \psi_{x} \theta d x .
\end{aligned}
$$

Adding together (3.14) and (3.16), we obtain

$$
F_{1}^{\prime}(t)+\delta F_{1}(t)=G_{1}(t) .
$$

Taking $\delta$ small enough such that

$$
\delta-2 \kappa \alpha<0, \quad 3 \delta-2 \lambda<0, \quad 1-\delta(\lambda-\delta)>0,
$$

we can render several terms of $G_{1}$ negative. Using the estimate (3.12), Lemma 3.1 and Young's inequality, we proceed with the following estimates

$$
\begin{aligned}
\left.\left|\int \theta\right| \psi\right|^{2} d x \mid & \leq\|\theta\|\|\mid \psi\|_{4}^{2} \leq \text { const }\|\theta\|\left\|\psi_{x}\right\|^{1 / 2}\|\psi\|^{3 / 2} \\
& \leq \text { const }\|\theta\|\left\|\psi_{x}\right\|^{1 / 2} \\
& \leq \epsilon_{1}\|\theta\|^{2}+\frac{\epsilon_{2}}{2}\left\|\psi_{x}\right\|^{2}+C .
\end{aligned}
$$


and

$$
\left.\left|2 \alpha \int \phi\right| \psi\right|^{2} d x \mid \leq \epsilon_{3}\|\phi\|^{2}+\frac{\epsilon_{2}}{2}\left\|\psi_{x}\right\|^{2}+C
$$

A fine point here is that the next integral couples the quantities we need to bound, as

$$
\left|\int \psi_{x} \theta d x\right| \leq\left\|\psi _ { x } \left|\||| \theta\| \leq \frac{\epsilon}{2}\left\|\psi_{x}\right\|^{2}+\frac{1}{2 \epsilon}\|\theta\|^{2} .\right.\right.
$$

Considering the above estimates, we wish to specify proper values for the arbitrary positive constants $\epsilon_{1}, \epsilon_{2}, \epsilon$, such that the following two inequalities hold simultaneously true

$$
\epsilon_{1}+\frac{1}{2 \epsilon} \leq-(3 \delta-2 \lambda), \epsilon_{2}+\frac{\epsilon}{2} \leq-(\delta-2 \kappa \alpha) .
$$

Let $\nu>0, \nu \neq \frac{1}{2}, \quad \tilde{\alpha}=-(3 \delta-2 \lambda)$ and $\tilde{\beta}=-(\delta-2 \kappa \alpha)$. Setting

$$
\epsilon_{1}=\frac{\tilde{\alpha}}{2 \nu}, \quad \epsilon_{2}=\frac{\tilde{\beta}}{2 \nu},
$$

we end up with the necessary condition

$$
\tilde{\alpha} \tilde{\beta} \geq \frac{\nu^{2}}{(2 \nu-1)^{2}} .
$$

Since $\tilde{\alpha}, \tilde{\beta}>0$ the inequality is always satisfied for sufficiently small $\nu$. Finally, taking $\epsilon_{3}$ small enough so that

$$
\epsilon_{3}<\delta(1-\delta(\lambda-\delta))
$$

we conclude that equation (3.17) gives

$$
F_{1}^{\prime}(t)+\delta F_{1}(t) \leq C
$$

An application of Gronwall's Inequality (Lemma 3.2) yields that there exists a positive constant $M_{1}$ such that, as $t \rightarrow \infty$, it holds

$$
\left(\|\psi(t)\|_{H_{0}^{1}}+\|\phi(t)\|_{H_{0}^{1}}+\|\theta(t)\|\right) \leq M_{1}
$$

Second Estimate: We continue with the a priori estimate in the space

$$
\left(H_{0}^{1}(\Omega) \cap H^{2}(\Omega)\right)^{2} \times H_{0}^{1}(\Omega) .
$$

Multiplying (3.5) by $\bar{\psi}_{x x}^{\prime}+\alpha \bar{\psi}_{x x}$, integrating over $\Omega$ and taking the real part, we get

$$
\frac{1}{2} \frac{d}{d t} \kappa\left\|\psi_{x x}\right\|^{2}+\kappa \alpha\left\|\psi_{x x}\right\|^{2}=\operatorname{Re} \int \phi \psi \bar{\psi}_{x x}^{\prime} d x+\alpha \operatorname{Re} \int \phi \psi \bar{\psi}_{x x} d x .
$$


Manipulating the right hand side in the following way

$$
\begin{aligned}
\operatorname{Re} \int \phi \psi \bar{\psi}^{\prime}{ }_{x x} d x= & +\frac{d}{d t} \operatorname{Re} \int \phi \psi \bar{\psi}_{x x} d x-\operatorname{Re} \int \theta \psi \bar{\psi}_{x x} d x \\
& +(\alpha+\delta) \operatorname{Re} \int \phi \psi \bar{\psi}_{x x} d x-\operatorname{Im} \int \phi^{2} \psi \bar{\psi}_{x x} d x
\end{aligned}
$$

equation (3.19) becomes

$$
\begin{aligned}
\frac{1}{2} \frac{d}{d t}\left(\kappa\left\|\psi_{x x}\right\|^{2}-2 \operatorname{Re} \int \phi \psi \bar{\psi}_{x x} d x\right) & +\kappa \alpha\left\|\psi_{x x}\right\|^{2} \\
-(2 \alpha+\delta) \operatorname{Re} \int \phi \psi \bar{\psi}_{x x} d x & =-\operatorname{Re} \int \theta \psi \bar{\psi}_{x x} d x-\operatorname{Im} \int \phi^{2} \psi \bar{\psi}_{x x} d x
\end{aligned}
$$

Next, multiply equation (3.7) by $-\theta_{x x}$ and integrate to get

$$
\begin{aligned}
\frac{1}{2} \frac{d}{d t}\left(\left\|\theta_{x}\right\|^{2}+\left\|\phi_{x x}\right\|^{2}+(1-\delta(\lambda-\delta))\left\|\phi_{x}\right\|^{2}\right) & +(\lambda-\delta)\left\|\theta_{x}\right\|^{2} \\
+\delta\left\|\phi_{x x}\right\|^{2}+\delta(1-\delta(\lambda-\delta))\left\|\phi_{x}\right\|^{2}= & -\operatorname{Re} \int \psi_{x x} \theta_{x} d x .
\end{aligned}
$$

We introduce the quantities

$$
F_{2}:=\kappa\left\|\psi_{x x}\right\|^{2}-2 R e \int \phi \psi \bar{\psi}_{x x} d x+\left\|\theta_{x}\right\|^{2}+\left\|\phi_{x x}\right\|^{2}+(1-\delta(\lambda-\delta))\left\|\phi_{x}\right\|^{2}
$$

and

$$
\begin{aligned}
G_{2}:= & +(\delta-2 \kappa \alpha)\left\|\psi_{x x}\right\|^{2}+(3 \delta-2 \lambda)\left\|\theta_{x}\right\|^{2}-\delta\left\|\phi_{x x}\right\|^{2} \\
& -\delta(1-\delta(\lambda-\delta))\left\|\phi_{x}\right\|^{2}+4 \alpha \operatorname{Re} \int \phi \psi \bar{\psi}_{x x} d x-2 \operatorname{Re} \int \psi_{x x} \theta_{x} d x \\
& -2 \operatorname{Re} \int \theta \psi \bar{\psi}_{x x} d x-2 \operatorname{Im} \int \phi^{2} \psi \bar{\psi}_{x x} d x .
\end{aligned}
$$

Adding together equation (3.20) and (3.21), we have

$$
F_{2}^{\prime}(t)+\delta F_{2}(t)=G_{2}(t), \quad t>0 .
$$

Following a procedure similar to the first estimate, we end up with

$$
F_{2}^{\prime}(t)+\delta F_{2}(t) \leq C, \quad t>0 .
$$

Finally, applying Gronwall's Inequality (Lemma 3.2), we conclude that there is a positive constant $M_{2}$ such that, for $t \rightarrow \infty$, it holds

$$
\left(\|\psi(t)\|_{H_{0}^{1} \cap H^{2}}+\|\phi(t)\|_{H_{0}^{1} \cap H^{2}}+\|\theta(t)\|_{H_{0}^{1}}\right) \leq M_{2} .
$$

We are ready to formulate and prove the main result of this section. 
Theorem 3.1 Assume that

$$
\left(\psi_{0}, \phi_{0}, \theta_{0}\right) \in\left(H_{0}^{1} \cap H^{2}(\Omega)\right)^{2} \times H_{0}^{1}(\Omega) .
$$

Then, there exists a unique solution for the system (3.5)-(3.9) such that

$$
\begin{aligned}
& \psi \in L^{\infty}\left(0, \infty ; H_{0}^{1}(\Omega) \cap H^{2}(\Omega)\right), \quad \psi_{t} \in L^{\infty}\left(0, \infty ; L^{2}(\Omega)\right), \\
& \phi \in L^{\infty}\left(0, \infty ; H_{0}^{1}(\Omega) \cap H^{2}(\Omega)\right), \quad \phi_{t} \in L^{\infty}\left(0, \infty ; H_{0}^{1}(\Omega)\right), \\
& \quad \phi_{t t} \in L^{\infty}\left(0, \infty ; L^{2}(\Omega)\right), \\
& \psi(x, 0)=\psi_{0}(x), \phi(x, 0)=\phi_{0}(x), \phi_{t}(x, 0)=\phi_{1}(x), x \in \Omega .
\end{aligned}
$$

Proof (Existence) The proof is based on the usual compactness arguments. We may consider the Galerkin system associated to (3.5)-(3.9). By similar computations, it is possible to verify that the estimates (3.18) and (3.22) hold also for the sequences of approximating solutions $\psi_{m}, \phi_{m}$, which can be constructed by using the complete orthonormal system, generated by the eigenfunctions of the operator $-\Delta, \mathrm{D}(-\Delta)=H_{0}^{1}(\Omega) \cap H^{2}(\Omega)$. We conclude that the sequences $\psi_{m}$ and $\phi_{m}$ are bounded in $L^{\infty}\left(0, \infty ; H_{0}^{1} \cap H^{2}(\Omega)\right)$ whereas the sequence $\theta_{m}$ is bounded in $L^{\infty}\left(0, \infty ; H_{0}^{1}(\Omega)\right)$. Therefore, we may extract weakly - $\star$ convergent subsequences, which are sufficient to pass to a limit $(\psi, \phi)$ in the Galerkin equations. The weak convergence process combined with elliptic regularity results shows that the pair $(\psi, \phi)$ is a solution of (3.5)-(3.9), sharing the properties (3.23).

(Uniqueness) Let $\left(\psi_{k}, \phi_{k}, \theta_{k}\right), k=1,2$ be two solutions in $X=\left(H_{0}^{1} \cap\right.$ $\left.H^{2}(\Omega)\right)^{2} \times H_{0}^{1}(\Omega)$, associated to the initial data $\left(\psi_{0 k}, \phi_{0 k}, \theta_{0 k}\right)$ and let $(\psi, \phi, \theta)=$ $\left(\psi_{1}-\psi_{2}, \phi_{1}-\phi_{2}, \theta_{1}-\theta_{2}\right),\left(\psi_{0}, \phi_{0}, \theta_{0}\right)=\left(\psi_{01}-\psi_{02}, \phi_{01}-\phi_{02}, \theta_{01}-\theta_{02}\right)$. Then, $(\psi, \phi, \theta)$ satisfies the following system

$$
\begin{array}{r}
i \psi^{\prime}+\kappa \psi_{x x}+i \alpha \psi=\phi_{1} \psi+\phi \psi_{2}, \\
\phi^{\prime}+\delta \phi=\theta, \\
\theta^{\prime}+(\lambda-\delta) \theta-\phi_{x x}+(1-\delta(\lambda-\delta)) \phi=-\operatorname{Re} \psi_{x},
\end{array}
$$

where the initial conditions are written as in (3.8).

Now, taking into account (3.12), (3.18) and (3.22), we can show that

$$
\begin{aligned}
\|\psi\|^{2}+\kappa\left\|\psi_{x}\right\|^{2}+\|\theta\|^{2} & +(1-\delta(\lambda-\delta))\|\phi\|^{2} \\
& +\kappa\left\|\psi_{x x}\right\|^{2}+\left\|\theta_{x}\right\|^{2}+\left\|\phi_{x x}\right\|^{2}+(2-\delta(\lambda-\delta))\left\|\phi_{x}\right\|^{2} \\
\leq & C\left(\left\|\psi_{0}\right\|^{2}+\left\|\psi_{0 x}\right\|^{2}+\left\|\theta_{0}\right\|^{2}+\left\|\phi_{0}\right\|^{2}+\left\|\psi_{0 x x}\right\|^{2}\right. \\
& \left.+\left\|\theta_{0 x}\right\|^{2}+\left\|\phi_{0 x x}\right\|^{2}+\left\|\phi_{0 x}\right\|^{2}\right) e^{C t}
\end{aligned}
$$

which proves the assertion. $\diamond$ 


\subsection{Energy Decay}

We return to the original system (3.1)-(3.4) and define the corresponding energy functional as

$$
E(t)=\frac{1}{2}\left(\|\psi\|^{2}+\kappa\left\|\psi_{x}\right\|^{2}+\int \phi|\psi|^{2} d x+\left\|\phi^{\prime}\right\|^{2}+\left\|\phi_{x}\right\|^{2}+\|\phi\|^{2}\right) .
$$

Note that, as in the Zakharov setup, the integral $\int \phi|\psi|^{2} d x$ determines the sign of the Hamiltonian. Nevertheless, this integral cannot possibly affect the asymptotic value of the energy, which remains positive, as indicated by the following result:

Lemma 3.3 Let the conditions of Theorem (3.1) be fulfilled. Further, assume that there is a positive constant $R$ such that $\|E(0)\| \leq R$. Then, there exists a $t^{*}>0$ such that, for every $t \geq t^{*}$, it holds $E(t)>0$.

Proof Under the assumptions for the initial data, Theorem (3.1) ensures that the energy $E(t)$ is well defined for all $t>0$. Now, from estimate (3.11), it turns out that

$$
\limsup _{t \rightarrow \infty}\|\psi(t)\|=0 .
$$

Therefore, for every $\epsilon_{0}>0$, there exists a $t^{*}>0$ such that,

$$
\|\psi(t)\|<\epsilon_{0}, \text { for all } t \geq t^{*} .
$$

We take the following estimate on the integral $\int \phi|\psi|^{2} d x$ as follows:

$$
\left.\left|\int \phi\right| \psi\right|^{2} d x\left|\leq\left\|\phi\left|\||| \psi\|_{4}^{2} \leq \tilde{C}\right||\phi|\right\|\right| \mid \psi_{x}\left\|^{1 / 2}\right\| \psi \|^{3 / 2}
$$

where $\tilde{C}$ is the positive constant induced by Lemma 3.1. Now, from relation (3.24), also using Poincaré's inequality $\|\psi\| \leq \lambda_{1}^{-1 / 2}\left\|\psi_{x}\right\|$, where $\lambda_{1}$ stands for the first eigenvalue of the Laplacian, the last estimate takes the form

$$
\begin{aligned}
\left.\left|\int \phi\right| \psi\right|^{2} d x \mid & \leq C\left(\lambda_{1}\right)\left\|\phi\left|\left\|\left|\psi_{x}\right|\right\|\right| \mid \psi\right\| \\
& \leq C\left\|\phi \left|\left\|\mid \psi_{x}\right\| \epsilon_{0}\right.\right. \\
& \leq \frac{1}{2}\|\phi\|^{2}+\frac{C^{2} \epsilon_{0}^{2}}{2}\left\|\psi_{x}\right\|^{2}, \text { for all } t \geq t^{*}
\end{aligned}
$$

Therefore,

$$
\int \phi|\psi|^{2} d x \geq-\frac{1}{2}\|\phi\|^{2}-\frac{C^{2} \epsilon_{0}^{2}}{2}\left\|\psi_{x}\right\|^{2}, \text { for all } t \geq t^{*}
$$


This estimate suggests that, for all $t \geq t^{*}$, it holds

$$
E(t) \geq \frac{1}{2}\left\{\|\psi\|^{2}+\left(\kappa-\frac{C^{2} \epsilon_{0}^{2}}{2}\right)\left\|\psi_{x}\right\|^{2}+\left\|\phi^{\prime}\right\|^{2}+\left\|\phi_{x}\right\|^{2}+\frac{1}{2}\|\phi\|^{2}\right\} .
$$

Choosing $\epsilon_{0}^{2}<\frac{2 \kappa}{C^{2}}$, justifies our claim. $\diamond$

Proceeding with the analysis, we multiply equation $(3.1)$ by $-\left(\bar{\psi}^{\prime}+\alpha \bar{\psi}\right)$. Integrating over $\Omega$ and taking the real part, yields

$$
\begin{aligned}
\frac{1}{2} \frac{d}{d t}\left(\kappa\left\|\psi_{x}\right\|^{2}+\int \phi|\psi|^{2} d x\right) & +\kappa \alpha\left\|\psi_{x}\right\|^{2} \\
& +\alpha \int \phi|\psi|^{2} d x=\frac{1}{2} \int \phi^{\prime}|\psi|^{2} d x .
\end{aligned}
$$

Next, we multiply equation (3.2) by $\phi^{\prime}$ and integrate to get

$$
\frac{1}{2} \frac{d}{d t}\left(\left\|\phi^{\prime}\right\|^{2}+\left\|\phi_{x}\right\|^{2}+\|\phi\|^{2}\right)+\lambda\left\|\phi^{\prime}\right\|^{2}=-\int R e \psi_{x} \phi^{\prime} d x .
$$

Adding together equations (3.10), (3.26) and (3.27) we obtain

$$
\begin{aligned}
E^{\prime}(t)+\alpha\|\psi\|^{2} & +\kappa \alpha\left\|\psi_{x}\right\|^{2}+\lambda\left\|\phi^{\prime}\right\|^{2} \\
& =-\alpha \int \phi|\psi|^{2} d x+\frac{1}{2} \int \phi^{\prime}|\psi|^{2} d x-\int \operatorname{Re} \psi_{x} \phi^{\prime} d x .
\end{aligned}
$$

We take estimates on the right side terms of (3.28) as follows:

$$
\left|\int R e \psi_{x} \phi^{\prime} d x\right| \leq \frac{1}{2 \epsilon_{1}}\left\|\psi_{x}\right\|^{2}+\frac{\epsilon_{1}}{2}\left\|\phi^{\prime}\right\|^{2}
$$

Next, as in Lemma (3.3), we have

$$
\begin{aligned}
\left.\left|\alpha \int \phi\right| \psi\right|^{2} d x \mid & \leq \alpha C\left(\lambda_{1}\right)\left\|\phi\left|\left\|\left|\psi_{x}\right|\right\|\right| \psi \mid\right. \\
& \leq \alpha \epsilon_{0} C\|\phi\|\left\|\psi_{x}\right\| \\
& \leq \frac{\epsilon}{2 \mu}\|\phi\|^{2}+\frac{\alpha^{2} \epsilon_{0}^{2} \hat{C}\left(\lambda_{1}, \mu\right)}{2 \epsilon}\left\|\psi_{x}\right\|^{2}, \text { for all } t \geq t^{*}
\end{aligned}
$$

where we have introduced the parameter $\mu>1$, which can be arbitrarily chosen. The last term is estimated in a similar fashion, this time using estimate (3.12)

$$
\begin{aligned}
\left.\left|\frac{1}{2} \int \phi^{\prime}\right| \psi\right|^{2} d x \mid & \leq C\left(\lambda_{1}\right)\left\|\phi ^ { \prime } \left|\left\||| \psi_{x}|\|||\psi|\right.\right.\right. \\
& \leq C R\left\|\phi ^ { \prime } \left|\left\|\mid \psi_{x}\right\|\right.\right. \\
& \leq \frac{R^{2} \epsilon_{1}}{2}\left\|\phi^{\prime}\right\|^{2}+\frac{1}{2 \epsilon_{1}}\left\|\psi_{x}\right\|^{2} .
\end{aligned}
$$


Note that the constant $C$ is incorporated into $R$. Collecting the estimates (3.29)(3.31), equation (3.28) for $t \geq t^{*}$ becomes

$$
E^{\prime}(t) \leq\left(\frac{1}{\epsilon_{1}}-\kappa \alpha+\frac{\alpha^{2} \epsilon_{0}^{2} \hat{C}}{2 \epsilon}\right)\left\|\psi_{x}\right\|^{2}+\left(\frac{\epsilon_{1}}{2}+\frac{R^{2} \epsilon_{1}}{2}-\lambda\right)\left\|\phi^{\prime}\right\|^{2}+\frac{\epsilon}{2 \mu}\|\phi\|^{2} .(3
$$

Following [12], for $\epsilon>0$, we introduce the perturbed energy

$$
E_{\text {pert }}(t)=E(t)+\epsilon p(t)
$$

where $p(t)=\|\psi(t)\|^{2}+\left(\phi^{\prime}(t), \phi(t)\right)$. Then,

$$
p^{\prime}(t)=2 \operatorname{Re}\left(\psi^{\prime}, \psi\right)+\left(\phi^{\prime \prime}, \phi\right)+\left\|\phi^{\prime}\right\|^{2} .
$$

Using equations (3.1) and (3.2), equation (3.34) becomes

$$
p^{\prime}(t)=-2 \alpha\|\psi\|^{2}-\left\|\phi_{x}\right\|^{2}-\|\phi\|^{2}+\left\|\phi^{\prime}\right\|^{2}-\lambda \int \phi^{\prime} \phi d x-\int \operatorname{Re} \psi_{x} \phi d x .
$$

Adding and subtracting several terms, also postulating

$$
N=\min \{4 \alpha, 1\}
$$

equation (3.35) gives

$$
\begin{aligned}
p^{\prime}(t) \leq & -N E(t)+\frac{\kappa}{2}\left\|\psi_{x}\right\|^{2}+\frac{3}{2}\left\|\phi^{\prime}\right\|^{2}-\frac{1}{2}\left\|\phi_{x}\right\|^{2}-\frac{1}{2}\|\phi\|^{2} \\
& +\frac{1}{2} \int \phi|\psi|^{2} d x-\lambda \int \phi^{\prime} \phi d x-\int R e \psi_{x} \phi d x .
\end{aligned}
$$

We proceed by estimating the integral terms as follows,

$$
\begin{aligned}
\left.\left|\frac{1}{2} \int \phi\right| \psi\right|^{2} d x \mid & \leq\left\|\phi\left|\left\|\left|\psi\left\|_{4}^{2} \leq \tilde{C}\right\| \phi\right|\right\|\right| \psi_{x}\right\|^{1 / 2}\|\psi\|^{3 / 2} \\
& \leq \frac{R \epsilon_{2}}{2}\|\phi\|^{2}+\frac{R}{2 \epsilon_{2}}\left\|\psi_{x}\right\|^{2} \\
\left|\lambda \int \phi^{\prime} \phi d x\right| & \leq \frac{\lambda^{2} \epsilon_{3}}{2}\left\|\phi^{\prime}\right\|^{2}+\frac{1}{2 \epsilon_{3}}\|\phi\|^{2} \\
\left|\int R e \psi_{x} \phi d x\right| & \leq \frac{\epsilon_{3}}{2}\left\|\psi_{x}\right\|^{2}+\frac{1}{2 \epsilon_{3}}\|\phi\|^{2} .
\end{aligned}
$$

Collecting (3.37)-(3.39), equation (3.36) becomes

$$
\begin{aligned}
p^{\prime}(t) \leq & -N E(t)+\left(\frac{\kappa}{2}+\frac{R}{2 \epsilon_{2}}+\frac{\epsilon_{3}}{2}\right)\left\|\psi_{x}\right\|^{2}+\left(\frac{3}{2}+\frac{\lambda^{2} \epsilon_{3}}{2}\right)\left\|\phi^{\prime}\right\|^{2} \\
& -\frac{1}{2}\left\|\phi_{x}\right\|^{2}+\left(\frac{R \epsilon_{2}}{2}+\frac{1}{\epsilon_{3}}-\frac{1}{2}\right)\|\phi\|^{2} .
\end{aligned}
$$


Now, differentiate (3.33) with respect to $t$ and use equations (3.32) and (3.40) to obtain

$$
\begin{aligned}
E_{\text {pert }}^{\prime}(t) \leq & -\epsilon N E(t)-\frac{\epsilon}{2}\left\|\phi_{x}\right\|^{2}+\left[\frac{1}{\epsilon_{1}}-\kappa \alpha+\frac{\alpha^{2} \epsilon_{0}^{2} \hat{C}}{2 \epsilon}+\epsilon\left(\frac{\kappa}{2}+\frac{R}{2 \epsilon_{2}}+\frac{\epsilon_{3}}{2}\right)\right]\left\|\psi_{x}\right\|^{2} \\
& +\left[\frac{\epsilon_{1}}{2}+\frac{R^{2} \epsilon_{1}}{2}-\lambda+\epsilon\left(\frac{3}{2}+\frac{\lambda^{2} \epsilon_{3}}{2}\right)\right]\left\|\phi^{\prime}\right\|^{2} \\
& +\epsilon\left[\frac{R \epsilon_{2}}{2}+\frac{1}{\epsilon_{3}}-\frac{1}{2}+\frac{1}{2 \mu}\right]\|\phi\|^{2} .
\end{aligned}
$$

We require that all expressions within the brackets are simultaneously nonpositive or zero. To achieve this, we introduce the auxiliary constant $\nu>0$. Then choosing the constants to be

$$
\epsilon_{2}=\frac{\nu}{R}, \quad \epsilon_{3}=\frac{1}{\nu}
$$

and setting the last expression equal to zero, we determine the value of $\nu$ as

$$
\nu=\frac{\mu-1}{3 \mu} .
$$

Consequently, the constants get the value

$$
\epsilon_{2}=\frac{\mu-1}{3 \mu R}, \quad \epsilon_{3}=\frac{3 \mu}{\mu-1} .
$$

Next, the implication that the second expression is nonpositive provides an upper bound for $\epsilon$ as

$$
\epsilon \leq \frac{2 \lambda-\left(1+R^{2}\right) \epsilon_{1}}{3+\lambda^{2} \epsilon_{3}}=: \hat{\epsilon_{1}} .
$$

Finally, we demand the first expression to be nonpositive. This leads to the quadratic inequality

$$
\epsilon^{2}\left(\frac{\kappa}{2}+\frac{R}{2 \epsilon_{2}}+\frac{\epsilon_{3}}{2}\right)+\epsilon\left(\frac{1}{\epsilon_{1}}-\kappa \alpha\right)+\frac{\alpha^{2} \epsilon_{0}^{2} \hat{C}}{2} \leq 0 .
$$

We are expecting two positive roots, one of which approaching zero, as $\epsilon_{0}$ does. Therefore, we have

$$
\hat{\epsilon_{2}}:=\frac{2\left(\kappa \alpha-\frac{1}{\epsilon_{1}}\right)}{\left(\kappa+\frac{R}{\epsilon_{2}}+\epsilon_{3}\right)} \geq \epsilon \gtrsim 0 .
$$


We purposely have not yet specified the value of $\epsilon_{1}$. In fact, this constant will be used to obtain the necessary conditions we need, by requiring that $\hat{\epsilon_{1}}$ and $\hat{\epsilon_{2}}$, in equations (3.42) and (3.43) respectively, have to be positive. Then, it should hold

$$
\epsilon_{1}>(\kappa \alpha)^{-1}
$$

and

$$
\epsilon_{1}<\frac{2 \lambda}{1+R^{2}}
$$

Combining relations (3.44) and (3.45) we get the conditions

$$
2 \lambda \alpha \kappa>1
$$

and

$$
R^{2}<2 \lambda \kappa \alpha-1
$$

which guarantee that

$$
E_{\text {pert }}^{\prime} \leq-\epsilon N E(t), \text { for all } t \geq t^{*} .
$$

We are ready to state the final result of this part.

Theorem 3.2 Suppose that for the parameters $\kappa, \lambda, \alpha$ condition (3.46) holds and there exists $R(\kappa, \lambda, \alpha)>0$, satisfying condition (3.47), such that $\|E(0)\| \leq R$. Then, the problem (3.1)-(3.4) manifests energy decay.

Proof It is readily shown that, for every $\epsilon>0$ and $t \geq t^{*}$, there exists a positive constant $C\left(\lambda_{1}\right)$, so that

$$
\left|E_{\text {pert }}(t)-E(t)\right| \leq \epsilon C\left(\lambda_{1}\right) E(t) .
$$

Now, from equations (3.48) and (3.49) we infer that, for $\epsilon \in\left(0, \epsilon_{0}\right]$, it holds

$$
E_{\text {pert }}(t) \leq 2 E(0) \exp \left(-\frac{\epsilon N t}{2}\right) \text {, for all } t \geq t^{*},
$$

where $\epsilon_{0}(\kappa, \lambda, \alpha)=\min \left\{\hat{\epsilon_{1}}, \hat{\epsilon_{2}}, \frac{1}{C\left(\lambda_{1}\right)}\right\} . \diamond$

Remark 3.1 The time $t^{*}$ introduced in the energy decay analysis has a specific physical meaning. This is the time so that the non-collisional integral $\int \phi|\psi|^{2} d x$ is absorbed by the collisional terms (see 3.25). Therefore, $t^{*}$ roughly signifies the time required by the collisional damping to smooth out the excessive difference of kinetic energies of resonant electrons and non-resonant ions (equipartition). Given standard reaction conditions, this time is of the order of $10^{-8}-10^{-6}$ seconds. 


\section{Part III: Physical Interpretation}

We write equations (2.14) and (2.26) in dimensionless form. For this, we introduce the dimensionless variables

$$
\hat{t}=A t, \hat{x}=B x, \hat{E}=\Gamma \tilde{E}, \hat{n}=\Delta n^{L},
$$

where $A, B, \Gamma, \Delta$ are positive constants. Now, equation (2.26) becomes

$$
i \frac{\partial \hat{E}}{\partial \hat{t}}+\frac{\gamma_{e} v_{t h, e}^{2}}{2 \omega_{u h}} \frac{B^{2}}{A} \frac{\partial^{2} \hat{E}}{\partial \hat{x}^{2}}+i \frac{\nu_{e i}}{2 A} \hat{E}=\frac{\omega_{p}^{2}}{2 \omega_{u h} n_{0}} \frac{1}{A \Delta} \hat{n} \hat{E}
$$

whereas equation (2.14) gives

$$
\frac{\partial^{2} \hat{n}}{\partial \hat{t}^{2}}-\gamma_{i} v_{t h, i}^{2} \frac{B^{2}}{A^{2}} \frac{\partial^{2} \hat{n}}{\partial \hat{x}^{2}}+\frac{\omega_{i}^{2}}{A^{2}} \hat{n}+\frac{\omega_{p}^{2}}{\omega_{e}^{2}} \frac{\nu_{e i}}{A} \frac{\partial \hat{n}}{\partial \hat{t}}=-\frac{e n_{0}}{m_{i}} \frac{B \Delta}{A^{2} \Gamma} \frac{\partial \hat{E}_{r}}{\partial \hat{x}} .
$$

In equation (4.2) we set

$$
\frac{\omega_{i}^{2}}{A^{2}}=1 \text { and } \gamma_{i} v_{t h, i}^{2} \frac{B^{2}}{A^{2}}=1
$$

from which we get

$$
A=\omega_{i} \text { and } B^{2}=\frac{\omega_{i}^{2}}{\gamma_{i} v_{t h, i}^{2}} .
$$

In this fashion, though not necessary, we can determine the expressions for $\Gamma$ and $\Delta$ as well. The normalized system of equations now reads

$$
\begin{aligned}
i \hat{E}_{t}+\kappa \hat{E}_{x x}+i \alpha \hat{E} & =\hat{n} \hat{E}, \\
\hat{n}_{t t}-\hat{n}_{x x}+\hat{n}+\lambda \hat{n}_{t} & =-\frac{\partial}{\partial x} \hat{E}_{r},
\end{aligned}
$$

with the parameters

$$
\kappa=\frac{\omega_{i}}{2 \omega_{u h}} \frac{\gamma_{e} v_{t h, e}^{2}}{\gamma_{i} v_{t h, i}^{2}}, \quad \alpha=\frac{\nu_{e i}}{2 \omega_{i}}, \quad \lambda=\frac{\omega_{p}^{2} \nu_{e i}}{\omega_{i} \omega_{e}^{2}} .
$$

Now, using standard relations (see [22]), we may express the parameters as functions of the plasma variables $n, T_{e}, T_{i}$ and the magnetic field $B_{0}$. We have $\left(T_{e}\right.$ in $\mathrm{keV}, n$ in $m^{-3}, B_{0}$ in Tesla)

$$
\begin{aligned}
\omega_{p}=56.4 n^{1 / 2}, & \omega_{i}=95.5 \cdot 10^{6} B_{0}, \\
\omega_{e}=17.6 \cdot 10^{10} B_{0}, & \nu_{e i}=1.6 \cdot 10^{-15} \frac{n}{T_{e}^{3 / 2}} .
\end{aligned}
$$


Now, the necessary condition (3.46) for the energy decay reads

$$
\frac{\omega_{p}^{2} \nu_{e i}^{2}}{2 \omega_{u h} \omega_{i} \omega_{e}^{2}} \frac{\gamma_{e} v_{t h, e}^{2}}{\gamma_{i} v_{t h, i}^{2}}>1 .
$$

To get a better understanding, we make the plausible assumption $\omega_{u h} \cong 2 \omega_{p}$, thus ending up with

$$
\frac{n^{5 / 2}}{T_{e}^{2} T_{i} B_{0}^{3}} \gtrsim 8 \cdot 10^{55}
$$

which establishes a threshold of the effectiveness of UHH in terms of the plasma variables and the magnetic field.

\section{Conclusions}

In this work we investigate a KGS-type evolution system, adapted to describe the UHH scheme for a magnetical confinement fusion device. The model focuses on Coulomb collisions, the fundamental mechanism of energy transfer between resonant electrons and non-resonant ions, thus producing bulk heating of the plasma. This is achieved by investigating the ion channel via the non-homogeneous polarization drift. A dc magnetic field is also considered, allowing for the modelling of perpendicular waves, peculiar to this heating scheme.

With respect to the methematical setup, the 1-D configuration is by no means restrictive, since the model effectively describes 3-D physical dynamics: dc magnetic field in the $\hat{z}$ direction, polarization drift in the $\hat{x}$ direction and $\vec{E} \times \vec{B}$ drift in the $\hat{y}$ direction. Further, the bounded domain manifests itself exclusively through Poincaré's e inequality. However, we would like to note, that possible generalisations, including the study of a similar model in a bounded or an unbounded domain and the generalisation of the results in more regular spaces, could possibly be a plan of future work.

The physical interpretation of the results ends up with a threshold of the effectiveness of UHH, involving the plasma variables, i.e., density, ion and electron temperatures as well as the magnetic field. Our result suggests that UHH is favoured by high-density conditions, such as in the very promising density-limit shots, where also the temperature assumes relatively low values.

Acknowledgements. We would like to thank the referee for fruitful suggestions (especially for the discussion concerning Lemma 3.3), which improved the original results and presentation of the original manuscript. This work was partially supported by a grant from the Thalis project No 65/1211 from the Committee of Fundamental Research at NTUA, Athens, Greece. 


\section{References}

[1] Biler P., Attractors for the system of Schrödinger and Klein-Gordon equations with Yukawa coupling, SIAM J. Math. Anal. 21, (1990), no. 5, 1190.

[2] Boling G., Yongsheng L., Attractor for dissipative Klein-Gordon-Schrödinger equations in $\mathbb{R}^{3}$, J. Differential Equations, 136, (1997), 356.

[3] Cavalcanti M.M., Cavalcanti V.N.D., Global existence and uniform decay for the coupled Klein-Gordon Schrödinger equations, NoDEA Nonlinear Differential Equations Appl., Birkhäuser 7, (2000), no. 3, 285.

[4] Bourgain J., On the Cauchy and invariant measure problem for the periodic Zakharov system, Duke Math. J. 76, (1994), no. 1, 175.

[5] Bourgain J., Colliander J., On wellposedness of the Zakharov system, Internat. Math. Res. Notices, no. 11, (1996), 515.

[6] Chen F., Plasma Physics and Controlled Fusion, Vol.I, Plenum Press, 1974.

[7] Colliander, J., Wellposedness for Zakharov systems with generalized nonlinearity, J. Differential Equations 148, (1998), no. 2, 351.

[8] Flahaut I., Attractors for the dissipative Zakharov system, Nonlinear Anal. 16, (1991), no. 8, 599.

[9] Ginibre J., Tsutsumi Y., Velo G., On the Cauchy problem for the Zakharov system, J. Funct. Anal. 151, (1997), no. 2, 384.

[10] Goubet O., Moise I., Attractor for dissipative Zakharov system, Nonlinear Anal. 31 (1998), no. 7, 823.

[11] Gross E. P., Krook M., Model for Collision Processes in Gases: SmallAmplitude Oscillations of Charged Two-Component Systems, Phys. Rev. 102, (1956), 593.

[12] Haraux A., Zuazua E., Decay estimates for some semilinear damped hyperbolic problems, Arch. Rational Mech. Anal. 100, (1988), no. 2, 191.

[13] Lu K., Wang B., Global attractors for the Klein-Gordon-Schrödinger equation in unbounded domains, J. Differential Equations 170, (2001), 281.

[14] Maingi R. et al., Density Limit Studies in DIII-D, J. Nucl. Mater. 266-269, (1999), 598.

[15] Masselin, Vincent A, result on the blow-up rate for the Zakharov system in dimension 3, SIAM J. Math. Anal. 33, (2001), no. 2, 440. 
[16] Nicholson D. R., Introduction to Plasma Theory, John Wiley and Sons, 1983.

[17] Pecher H., Global well-posedness below energy space for the 1-dimensional Zakharov system, Internat. Math. Res. Notices, (2001), no. 19, 1027.

[18] Sulem, C., Sulem, P.L., The Nonlinear Schrödinger Equation, Applied Mathematical Sciences Vol. 139, Springer-Verlag, 1999.

[19] Takaoka H., Well-posedness for the Zakharov system with the periodic boundary condition, Differential Integral Equations 12, (1999), no. 6, 789.

[20] Temam R., Infinite-Dimensional Dynamical Systems in Mechanics and Physics, (2nd Edition), Appl. Math. Sc., 68, Springer-Verlag, New York, (1997).

[21] Wesner F., Summer Univ. for Plasma Physics, Max-Planck Institut fuer Plasma Physics, 1995.

[22] Wesson J., Tokamaks, Clarendon Press, Oxford, 1987.

[23] Zakharov V. E., Collapse of Langmuir Waves, Sov. Phys. JETP 35, (1972), 908.

[24] Zeidler E., Nonlinear Functional Analysis and its Applications, Vols I, II, (Fixed Point Theorems, Monotone Operators), Springer-Verlag, Berlin, 1990.

${ }^{\dagger}$ Department of Statistics and Actuarial Science

University of the Aegean,

Karlovassi, 83200 Samos, GREECE

E-mail address: karan@aegean.gr, nkaraha@math.ntua.gr

$\ddagger$ Department of Mathematics

National Technical University

Zografou Campus 15780 Athens, Hellas

E-mail address: nikolas@central.ntua.gr 